\title{
KPNA2 is a promising biomarker candidate for esophageal squamous cell carcinoma and correlates with cell proliferation
}

\author{
SHOUZHI MA $^{1}$ and XIAOHANG $\mathrm{ZHAO}^{1,2}$ \\ ${ }^{1}$ State Key Laboratory of Molecular Oncology, Cancer Institute and Hospital, \\ Chinese Academy of Medical Sciences and Peking Union Medical College, Beijing 100021; \\ ${ }^{2}$ Center of Basic Medical Sciences, Navy General Hospital, Beijing 100048, P.R. China
}

Received April 8, 2014; Accepted July 9, 2014

DOI: $10.3892 /$ or.2014.3381

\begin{abstract}
Esophageal squamous cell carcinoma (ESCC) is one of the most lethal malignant cancers worldwide, with a poor 5-year prognosis. Karyopherin $\alpha 2$ (KPNA2) is a nuclear membrane protein that mediates nucleus-to-cytoplasm shuttling. Its expression is elevated in multiple forms of cancer, and it can be secreted into the serum. However, the concentration of KPNA2 in serum from ESCC patients and the role of KPNA2 in ESCC cells remains unclear. The aim of the present study was to determine the concentration of KPNA2 in serum from ESCC patients and to investigate the effect of KPNA2 silencing on ESCC cell proliferation. KPNA2 protein expression was detected at the tissue level by immunohistochemistry, in cell lines by western blotting and at the serum level by enzyme linked immunosorbent assay (ELISA). Cell proliferation was determined by cell growth curve and colony formation assay. Stages of the cell cycle were analyzed by flow cytometry. The effect of KPNA2 knockdown on E2F1 translocation was determined by subcellular fractionation. KPNA2 was overexpressed in both ESCC tissues and cell lines compared with controls. The concentration of KPNA2 in serum from ESCC patients was significantly higher than that from healthy controls. The AUC was determined to be 0.804 . The sensitivity and specificity of the assay were 76.7 and $75.0 \%$, respectively. To determine the significance of KPNA2 function, small interfering RNA (siRNA) against KPNA2 was used to knock down KPNA2 levels in the ESCC using siRNA in the Kyse510 cell line. KPNA2 siRNA inhibited Kyse510 cell proliferation and colony formation ability and induced a G2/M phase arrest. The nuclear translocation of E2F1 was also reduced in siRNA-treated Kyse510 cells. The KPNA2 protein levels were high in ESCC tumors, and siRNA against KPNA2
\end{abstract}

Correspondence to: Professor Xiao-Hang Zhao, Center of Basic Medical Sciences, Navy General Hospital and State Key Laboratory of Molecular Oncology, Cancer Institute and Hospital, CAMS and PUMC, Beijing 100021, P.R. China

E-mail: zxhtao@yeah.net

Key words: KPNA2, esophageal squamous cell carcinoma, tumor biomarkers, proliferation, cell cycle could inhibit the growth of ESCC cells, suggesting it may be a new potent marker and therapeutic target for ESCC.

\section{Introduction}

Esophageal cancer is the 5th most common cause of cancerrelated mortality in males worldwide and the 8th most common in females (1). Esophageal squamous cell carcinoma (ESCC) is a major histopathologic subtype of esophageal cancer. The prognosis of patients with ESCC is poor, and the 5 -year survival rate is $<30 \%$ (2). Identifying new markers and therapeutic targets may help in the early detection of ESCC and may reverse the malignant phenotype, thus improving the prognosis.

Karyopherin $\alpha 2$ (KPNA2) is one of the seven family members of karyopherin $\alpha$ (3). These proteins mediate the nucleocytoplasmic transport through large nuclear pore complexes. With the help of KPNA2, macromolecules $>40 \mathrm{kDa}$ can be shuttled between the cytoplasm and nucleus (4). During nucleocytoplasmic transport, karyopherin $\alpha$ can form heterodimers with karyopherin $\beta-1$ to generate the nuclear transport complex. As part of the complex, KPNA2 can recognize cargo proteins via their nuclear localization signal and act as an adaptor. Karyopherin $\beta-1$ then facilitates protein docking to and translocation through the nuclear pore complexes. After entering the nucleus, karyopherin $\alpha$ and karyopherin $\beta-1$ can dissociate from the complex and bind RanGTP, respectively. They are then recycled back into the cytoplasm $(4,5)$.

Deregulation of the cellular transport machinery often occurs in tumors. In accordance with this phenomenon, KPNA2 expression has been shown to be elevated in a variety of tumor tissues, including breast (6-8) and ovarian cancer (9), melanoma (10), cervical (11), lung (12), brain (13), prostate (14), liver (15), bladder (16) and esophageal cancer (17). Notably, it has been shown that KPNA2 expression is higher in both tissue and serum in lung cancer (12), suggesting that KPNA2 can be secreted into the serum. Since the overexpression of KPNA2 is a common phenomenon in different types of cancer, it is possible that KPNA2 serum levels may also be upregulated in other types of cancer. However, with the exception of lung cancer, similar results have not been reported until now.

In ESCC, KPNA2 has been shown to be upregulated in the tumor tissue (17). The high expression of KPNA2 correlates 
with a poor prognosis. However, the expression of KPNA2 in serum and the function of KPNA2 in ESCC cells remains unclear. In the present study, we observed high expression of KPNA2 in tissues, cell lines and serum from patients with ESCC. Knockdown of KPNA2 in an ESCC cell line inhibited cell proliferation by inducing $\mathrm{G} 2 / \mathrm{M}$ phase cell cycle arrest and attenuated E2F1 nuclear translocation. These results indicate that KPNA2 may be a potent marker and therapeutic target of ESCC.

\section{Materials and methods}

Chemicals. Dulbecco's modified Eagle's medium (DMEM) and RPMI-1640 culture media were purchased from Invitrogen (Carlsbad, CA, USA). Fetal bovine serum (FBS) was obtained from HyClone (Logan, UT, USA). Goat anti-KPNA2 (sc-6917) and rabbit anti-E2F1 primary antibody (sc-193) were obtained from Santa Cruz Biotechnology (Santa Cruz, CA, USA). Goat anti-laminB (ab16048) primary antibody was purchased from Abcam (Cambridge, UK). Mouse anti- $\beta$-actin primary antibody (A-5316) was obtained from Sigma (Santa Clara, CA, USA).

Clinical specimens. Tissue specimens were purchased as a microarray (Outdo Biotech Co., Shanghai, China). The median age of the patients ( 71 males, 22 females) from whom the ESCC tissues were collected for immunochemistry was 64.2 years (range, 49-78 years). Surgical tissue specimens were collected from ESCC patients with their informed consent and approval from the Institutional Review Board of the Cancer Institute and Hospital of Chinese Academy of Medical Sciences (Beijing, China). Serum samples were collected from 86 ESCC patients (70 males and 16 females; mean age, 59.5 years; range, 39-76 years) and 60 healthy controls (43 males and 17 females; mean age, 53 years; range, 26-76 years), who all provided informed consent. Pathological diagnosis was conducted independently by two senior pathologists. Biochemistry tests and routine blood tests were performed for the healthy controls, and the results were within the normal ranges. Sample preparation was previously described (18).

Cell culture. Kyse30, Kyse140, Kyse150, Kyse170, Kyse410 and Kyse 510 cells were donated by Dr Y. Shimada. These cells were cultured in RPMI-1640 medium supplemented with $10 \%$ FBS, $1 \% 100 \mathrm{U} / \mathrm{ml}$ penicillin and $100 \mathrm{mg} / \mathrm{ml}$ streptomycin. WHCO1 cells were grown in DMEM supplemented with $10 \%$ FBS. All cells were maintained at $37^{\circ} \mathrm{C}$ in $5 \% \mathrm{CO}_{2}$.

Immunohistochemistry. For immunohistochemical staining, multiple tissue arrays (MTAs) were incubated with goat anti-KPNA2 polyclonal antibody. After washing with $1 \mathrm{X}$ phosphate-buffered saline (PBS), slides were reacted with the biotin-labeled second antibody and then visualized using an ultrasensitive streptavidin-peroxidase system (ZSGB Biotechnology, Beijing, China). Immunostaining was scored as follows: 0 , negative; 1 , weak; 2 , moderate; 3 , strong staining. The percentage of KPNA2 staining area was graded as 0 , no positive staining; $1,<5 \% ; 2,5-25 \% ; 3,50-75 \%$; or $4,>75 \%$. The staining index was calculated as the product of staining intensity and staining area, ranging from 0 to 12 .
Enzyme linked immunosorbent assay (ELISA). The KPNA2 levels in human serum were measured using a commercially available ELISA kit (USCN Life Science Inc., Wuhan, Hubei, China). The ELISA was performed according to the manufacturer's instructions. Briefly, $100 \mu \mathrm{l}$ of diluted serum samples (at 1:500 dilution) was added to each well and incubated at $37^{\circ} \mathrm{C}$ for $2 \mathrm{~h}$. Then, $100 \mu \mathrm{l}$ reagent A was added for an additional hour. After 5 washes, reagent B was added for $30 \mathrm{~min}$. The amount of proteins was determined by adding TMB substrate, and the plate was incubated at $37^{\circ} \mathrm{C}$ to allow for color development. The absorbance was measured at $450 \mathrm{~nm}$ using a Model 680 microplate reader (Bio-Rad Laboratory Inc., Hercules, CA, USA).

Western blotting. Approximately $15 \mu \mathrm{g}$ protein lysate was separated on SDS-PAGE gels and transferred to PVDF membranes (GE Healthcare, Piscataway, NJ, USA). After blocking, the membranes were incubated with primary antibodies and developed using an ECL system (Applygen Technologies Inc., Beijing, China). LaminB and $\beta$-actin were used as loading controls.

Small interfering RNA (siRNA) transfection. siRNAs were synthesized by Invitrogen Co. (Shanghai, China), and the target sequences were: siRNA-1, 5'-GCUCCUGCAUCAUGAU GAU-3'; siRNA-2, 5'-GUGGCUACUUACGUAAUCU-3'; negative control, 5'-UUCUCCGAACGUGUCACGU-3'. Transient transfection was performed using Lipofectamine 2000 (Life Technologies, Carlsbad, CA, USA), according to the manufacturer's protocol. Briefly, the cells were seeded in 6-well plates and allowed to grow for at least $20 \mathrm{~h}$. Then, $100 \mathrm{nmol}$ siRNA was added to each well. After a 6-h transfection, the siRNAs were replaced by adding fresh medium. Western blotting was used to confirm the knockdown efficiency.

Cell proliferation analysis. Cell proliferation was assessed using the WST- 8 method based on the metabolic reduction of the monosodium salt 2-(2-methoxy-4-nitrophenyl)-3-(4nitrophenyl)-5-(2,4-sulfophenyl)-2H tetrazolium. Three thousand cells were seeded in 96-well plates and cultured overnight. The cells were transfected with mock or siRNAs by Lipofectamine 2000. Then, $10 \mu \mathrm{l}$ WST- 8 solution was added to each well and incubated for $2 \mathrm{~h}$. The plates were read on a Model 680 microplate reader at $450 \mathrm{~nm}$. Colony formation assays were performed as previously described (19).

Flow cytometry. Cells were trypsinized, fixed with ethanol and analyzed by flow cytometry (LSR II; BD Biosciences, Lexington, KY, USA). Briefly, fixed cells were washed twice with ice-cold PBS. RNAse $(20 \mathrm{mg} / \mathrm{ml})$ and PI $(50 \mathrm{mg} / \mathrm{ml})$ were added and incubated for $30 \mathrm{~min}$ at $37^{\circ} \mathrm{C}$ in the dark. Ten thousand cells per sample were subjected to flow cytometry. The results were analyzed using the FlowJo software (Tree Star Inc., Ashland, OR, USA).

Subcellular protein fractionation. Subcellular protein fractionation was performed using a ProteoExtract Subcellular Proteome Extraction kit (Calbiochem, La Jolla, CA, USA), according to the manufacturer's instructions. Briefly, adherent cells were digested with trypsin and washed twice with PBS. 


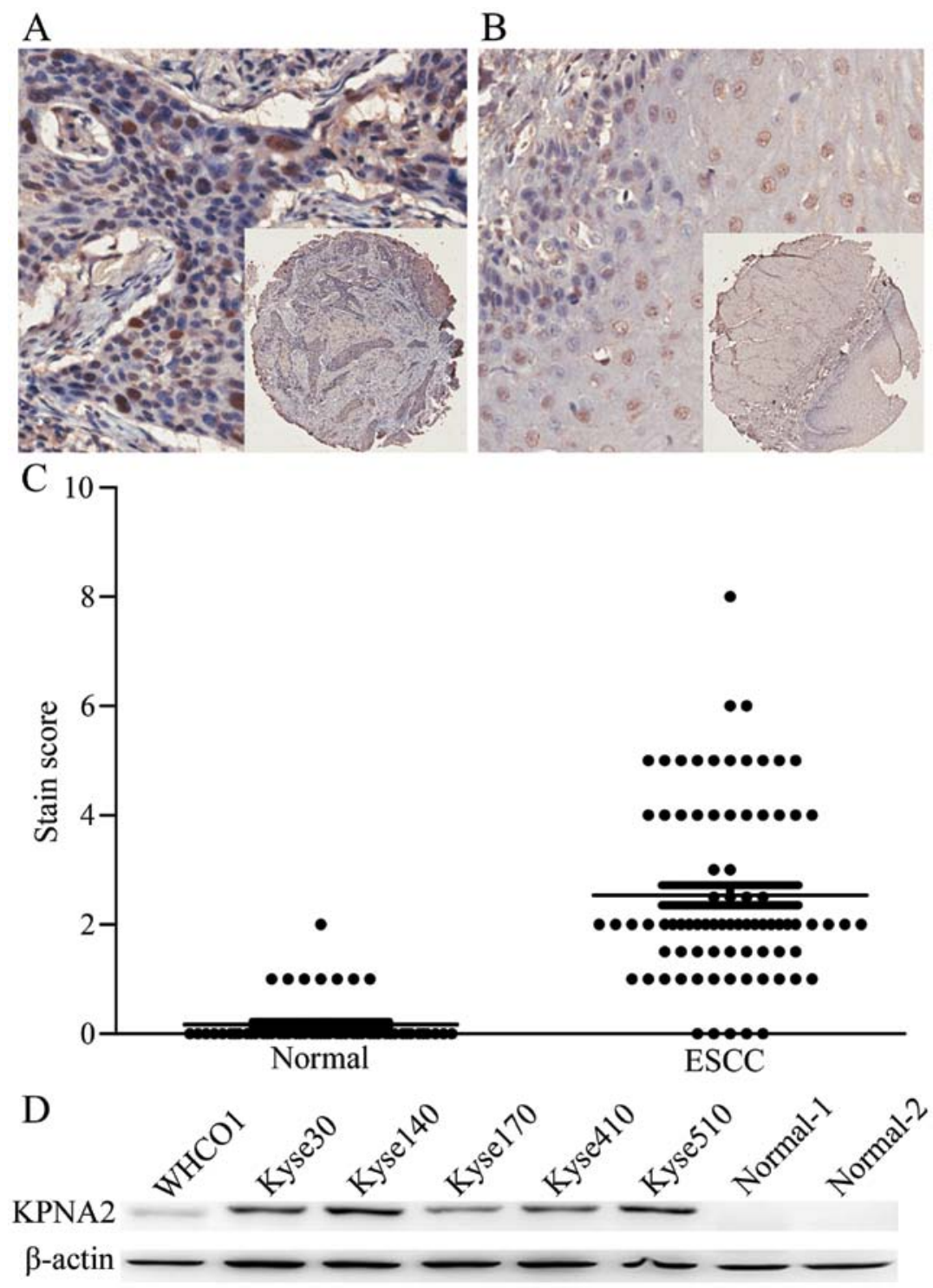

Figure 1. Expression of KPNA2 in human ESCC tissues and cell lines. (A) Representative immunohistochemical staining of KPNA2 in ESCC tissues. Positive signals were detected in the nuclei of the tumor cells. (B) Representative immunohistochemical staining of KPNA2 in paired normal esophageal tissues. Nuclei were only stained weakly in control tissues. (C) Immunohistochemical staining scores of ESCC and paired adjacent normal esophageal tissues stained with the KPNA2 antibody. KPNA2 was significantly upregulated in ESCC tissues (Student's t-test, P<0.0001). (D) Western blot analysis of KPNA2 in 6 ESCC cell lines and 2 adjacent normal esophageal tissues. KPNA2, karyopherin $\alpha 2$; ESCC, esophageal squamous cell carcinoma.

Extraction buffers I, II and III were sequentially added to generate different fractions. Fraction 1 includes the cytosolic fraction, fraction 2 includes the membrane fraction, and fraction 3 includes the nuclear fraction.

Statistical analysis. All statistical comparisons were performed using the SPSS 16.0 software (SPSS Inc., Chicago, IL, USA). Student's t-test was used to determine the statistical significance of the results. $\mathrm{P}<0.05$ was considered to indicate a statistically significant difference.

\section{Results}

Expression of KPNA2 in ESCC tissues and cell lines. The expression of KPNA2 in ESCC tissues was examined by immunohistochemistry using tissue microarray. The results showed that KPNA2 was predominantly localized in the nuclei. Strong staining was observed in ESCC tissues, while only weak staining was observed in normal esophageal tissues (Fig. 1A and B). We semi-quantitated the staining pattern of KPNA2 in ESCC and paired normal esophageal tissues. The immunohistochemistry scores of KPNA2 ranged from 0 to 8 (median=2) in ESCC patients and from 0 to 2 (median=0) in normal esophageal tissues. KPNA2 staining was significantly higher in ESCC patients than in healthy controls (Fig. 1C, Student's t-test, $\mathrm{P}<0.0001$ ).

We next examined the KPNA2 expression in the ESCC cell lines. All 6 ESCC cell lines expressed KPNA2 at different levels (Fig. 1D). KPNA2 was detected with a strong signal in Kyse140 and Kyse510 cells, a moderate signal in Kyse30 and Kyse 410 cells and a weak signal in WHCO1 and Kyse170 cells. In normal human adjacent esophageal tissues, no obvious KPNA2 signal was detected. These results show that KPNA2 is also upregulated in ESCC cell lines. 

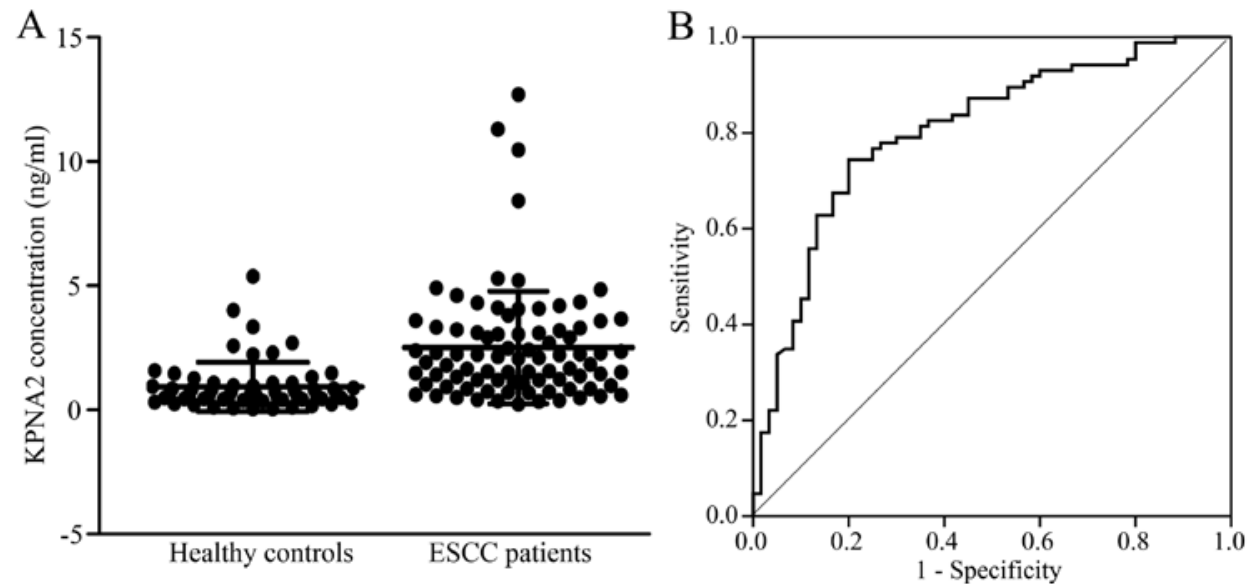

Figure 2. KPNA2 levels in serum from ESCC patients. (A) Serum levels of KPNA2 were measured in ESCC patients $(\mathrm{n}=86)$ and healthy volunteers $(\mathrm{n}=60)$ using ELISA. Serum levels of KPNA2 were significantly higher in the ESCC patients ( $\mathrm{P}<0.0001$, Mann-Whitney U test). (B) ROC curve analysis of diagnosis capacity of KPNA2 with SPSS. The area under curve (AUC) was 0.804 (95\% CI, 0.731-0.877). KPNA2, karyopherin $\alpha$ 2; ESCC, esophageal squamous cell carcinoma; CI, confidence interval.

A

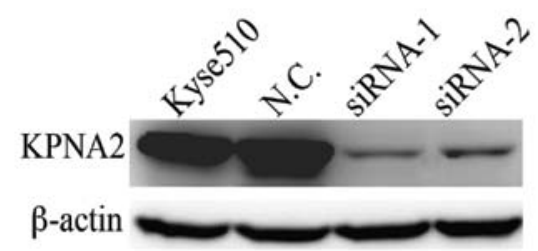

$\mathrm{C}$

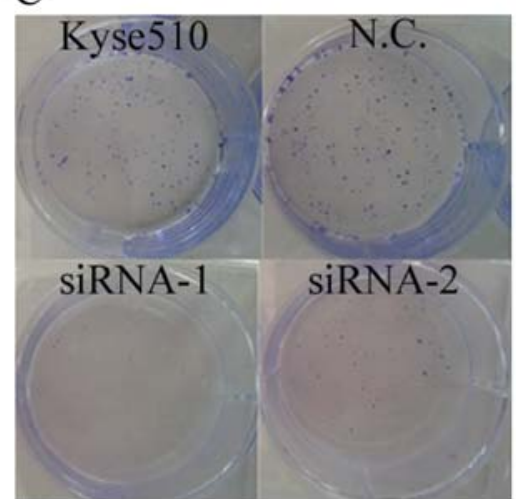

B

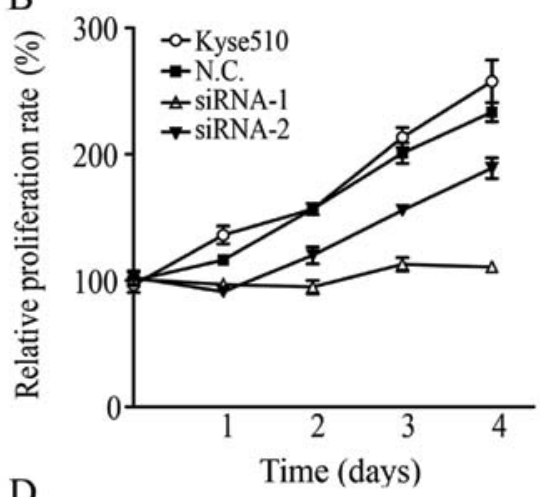

D

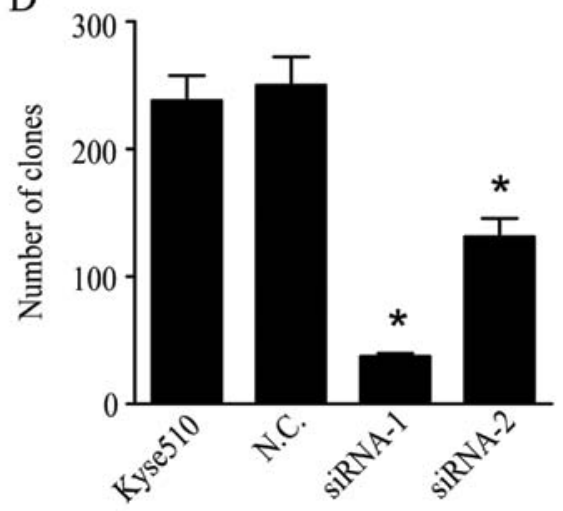

Figure 3. Effect of KPNA2 knockdown on Kyse510 cell proliferation and survival. (A) Analysis of the efficiency of KPNA2 knockdown in Kyse510 cells. Kyse 510 cells were transfected with the indicated siRNAs or Lipofectamine 2000 only (mock). Proteins were extracted $48 \mathrm{~h}$ later and subjected to western blotting. (B) WST-8 assays of mock or siRNA-transfected Kyse510 cells. Kyse510 cells $(3,000)$ were plated and grown, and the absorbance was analyzed every day. (C) Colony formation analysis of mock or siRNA-transfected Kyse510 cells. Mock or transfected cells (100) were seeded and grown for 10 days. Clones were fixed with ethanol and stained with crystal violet. (D) The number of clones was counted. (Student's t-test, "P<0.05). KPNA2, karyopherin $\alpha 2$; siRNA, small interfering RNA.

KPNA2 serum levels are higher in ESCC patients. We assessed the serum levels of KPNA2 in ESCC patients $(n=86)$ and healthy controls $(n=60)$ by ELISA. The serum concentrations of KPNA2 ranged from $0.19 \mu \mathrm{g} / \mathrm{ml}$ to $12.67 \mu \mathrm{g} / \mathrm{ml}$ (median $=1.95 \mu \mathrm{g} / \mathrm{ml}$ ) in ESCC patients and from $0 \mu \mathrm{g} / \mathrm{ml}$ to $5.34 \mu \mathrm{g} / \mathrm{ml}$ (median=0.58 $\mu \mathrm{g} / \mathrm{ml}$ ) in healthy controls (Fig. 2A). The KPNA2 levels were significantly higher in ESCC patients compared to healthy controls (Mann-Whitney U test, $\mathrm{P}<0.0001)$. The area under curve (AUC) was determined to be 0.804 [95\% confidence interval (CI), 0.731-0.877] for KPNA2 (Fig. 2B). When $0.96 \mu \mathrm{g} / \mathrm{ml}$ was used as the cut-off value, the sensitivity and specificity of serum KPNA2 was 76.7 and $75.0 \%$, respectively. These results suggest that KPNA2 may be a useful serum marker for ESCC. 

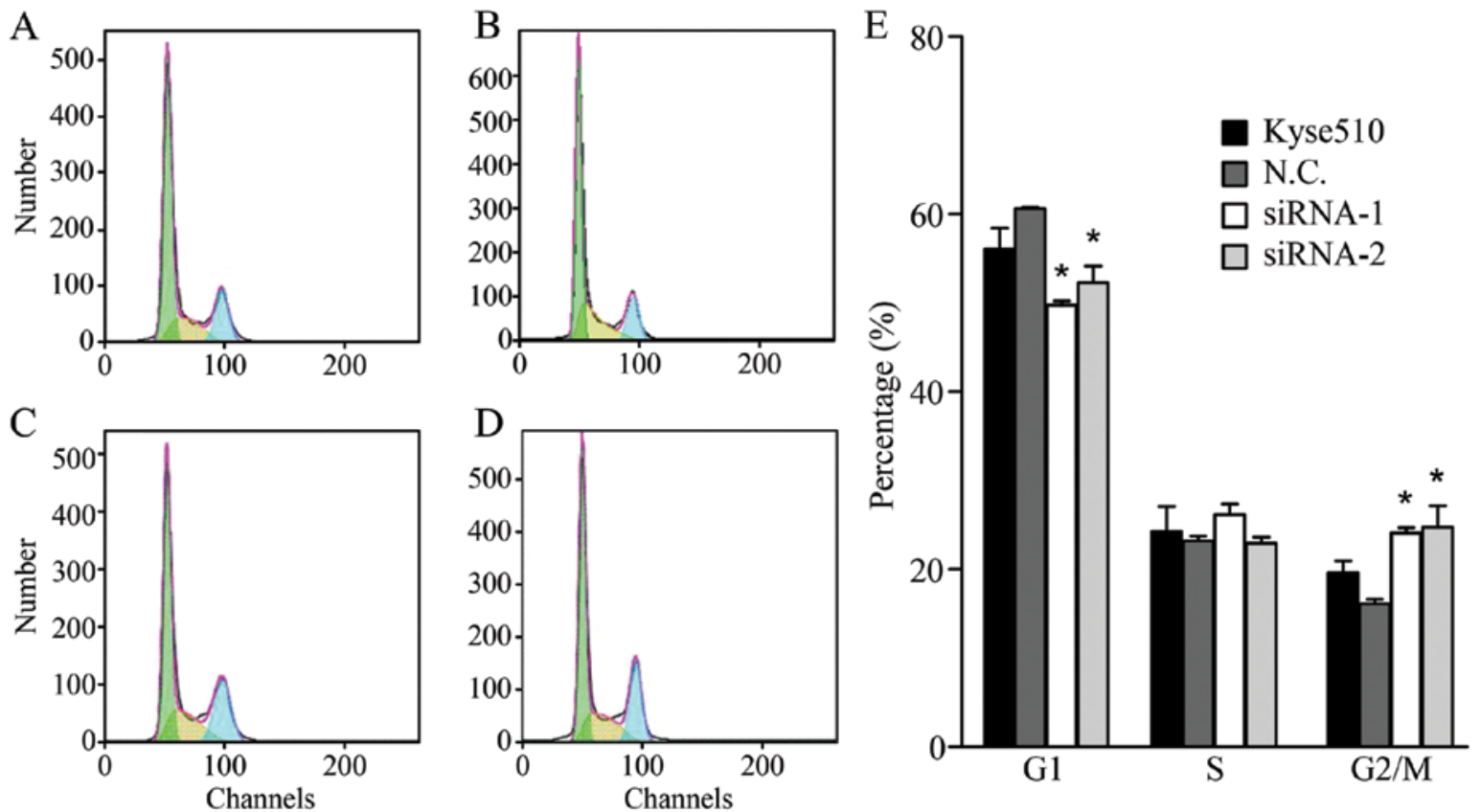

Figure 4. The cell cycle of Kyse510 cells was tested by flow cytometry (FCM). FCM analysis of the Kyse510 cells transfected with mock or siRNA. (A) Mock, (B) negative control, (C) siRNA-1, (D) siRNA-2. (E) Cell cycle distribution of Kyse510 cells. The percentage of cells in G1 phase were significantly reduced, while the percentage of G2/M phase cells was significantly increased in KPNA2 siRNA-transfected cells compared with control cells. (Student's t-test, $\left.{ }^{*} \mathrm{P}<0.05\right)$. siRNA, small interfering RNA; KPNA2, karyopherin $\alpha 2$.

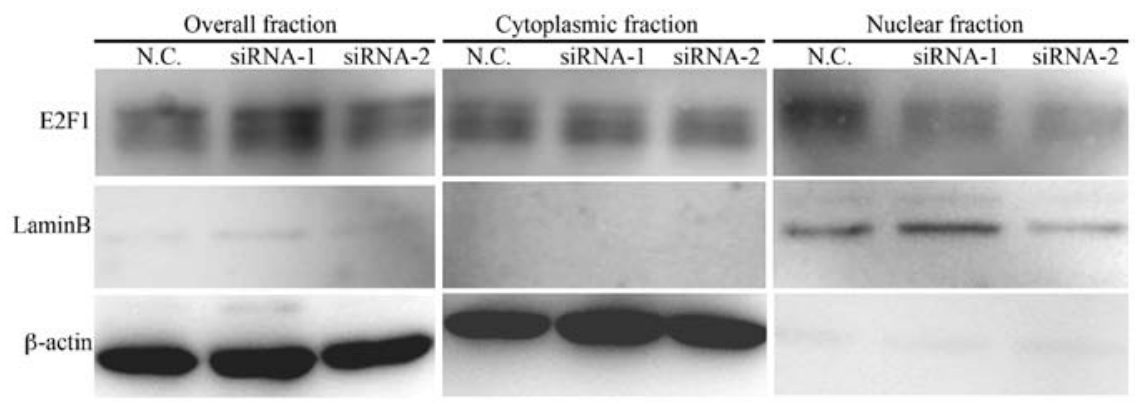

Figure 5. Effect of KPNA2 knockdown on E2F1 nuclear translocation. Subcellular proteins were extracted using a ProteoExtract Subcellular Proteome Extraction kit. Fractionation efficiency was determined by a western blot analysis of laminB and $\beta$-actin. The nuclear localization of E2F1 was reduced in KPNA2 knockdown cells compared with scramble siRNA-transfected cells. siRNA, small interfering RNA; KPNA2, karyopherin $\alpha 2$.

Knockdown of KPNA2 inhibits ESCC cell proliferation. To investigate the role of KPNA2 in human ESCC cells, we knocked down the KPNA2 expression with siRNA in Kyse510 cells. We used two siRNA sequences targeting different regions of KPNA2 mRNA and determined the efficiency of knockdown by western blotting. Both siRNAs significantly suppressed KPNA2 expression, and siRNA-1 showed higher knockdown efficiency than did siRNA-2 (Fig. 3A). A WST-8 assay showed that siRNA-1 almost completely abrogated Kyse510 cell growth and that siRNA-2 showed a moderate effect, whereas scramble siRNA exhibited no effect on ESCC cell proliferation (Fig. 3B). The colony formation assays showed that both siRNAs significantly reduced the clonogenicity of Kyse510 cells (Fig. 3C and D).

Knockdown of KPNA2 induces G2/M phase arrest. We assessed the effect of KPNA2 knockdown on cell cycle progression by flow cytometry. The results showed that the silencing of KPNA2 significantly decreased the percentage of cells in G0/G1 and increased the percentage of cells in the G2/M phase of the cell cycle (Fig. 4). The percentage of cells at S phase did not change. These results clearly show that the knockdown of KPNA2 induces a G2/M phase arrest in ESCC cells.

Knockdown of KPNA2 attenuates E2F1 nuclear translocation. E2F1 is a central regulator of proliferation and cell cycle progression and has been shown to be a target protein of KPNA2. We examined the expression of E2F1 in KPNA2 knockdown cells by subcellular fractionation. The fractionation efficiency was verified by a western blot analysis of laminB and $\beta$-actin in the cytoplasmic and nuclear fractions. The expression level of E2F1 was reduced in the nuclear fraction of KPNA2 knockdown ESCC cells compared with the scramble siRNA-transfected cells (Fig. 5). The results show that E2F1 nuclear translocation is inhibited in ESCC cells following KPNA2 knockdown. 


\section{Discussion}

In the present study, we reported that KPNA2 is upregulated in ESCC tissues, cell lines and sera. The knockdown of KPNA2 in ESCC cells inhibited cell proliferation and survival and induced G2/M cell cycle arrest. These effects may be partially mediated by reducing E2F1 nuclear translocation.

KPNA2 has been shown to be upregulated in various types of cancer. Our results showed that KPNA2 was higher in ESCC tissues, cell lines and serum. The increased expression of KPNA2 may be due to the altered transcriptional activity of tumors. Indeed, the upregulation of KPNA2 at the transcriptional level has been detected in cervical cancer (20). Additionally, its promoter activity has been studied extensively. The promoter region is located -80 to $-24 \mathrm{bp}$ upstream of the KPNA2 gene locus. In this region, there is a highly conserved binding site for E2F transcription factors. It was previously suggested that KPNA2 expression is initiated by E2F transcription factors. Notably, the nuclear import of E2F1 is also mediated by KPNA2 (21). This finding raised the possibility that a positive feedback loop may exist. In that feedback loop, E2F1 could increase the expression of KPNA2 and, in turn, KPNA2 increases the nuclear accumulation of E2F1. The expression of KPNA2 is also regulated by the cell cycle. The highest expression of KPNA2 occurs during the G2/M phase $(22,23)$.

Currently, ESCC diagnosis is predominantly made using cytological methods. The identification of useful ESCC serum markers remains limited. Our laboratory has demonstrated that the serum levels of GRO $\beta$ are elevated in ESCC patients (24). However, the use of only a single marker may be inefficient due to low sensitivity or specificity. Therefore, combining several markers as a panel could potentially provide better results. Here, we showed that KPNA2 is higher in serum from ESCC patients. We propose that KPNA2 could be used as a single or combined biomarker for the diagnosis of ESCC.

The effect of KPNA2 in tumor cell survival is controversial. KPNA2 has been shown to be involved in the proliferation of lung (12), liver (15) and prostate cancer (14), while no effect on proliferation has been observed in cervical cancer $(11,25)$. Our results showed that the knockdown of KPNA2 expression attenuated tumor cell proliferation in ESCC cells. This finding is consistent with phenomena observed in lung, liver and prostate cancer. These previous studies indicated that KPNA2 was a potential oncogene that functioned in sustaining cell proliferation. Since KPNA2 was upregulated in ESCC tissues and since reducing KPNA2 levels could attenuate cell proliferation, KPNA2 may be a potential therapeutic target of ESCC.

Either G1/S or G2/M cell cycle arrest can be induced by KPNA2 knockdown, depending on the cell context $(21,26)$. Our results showed that KPNA2 knockdown induced G2/M cell cycle arrest in ESCC cells. The synthesis of the proteins required for mitosis occurs during the G2/M phase. Notably, $\mathrm{E} 2 \mathrm{~F} 1$ or its homolog protein $\mathrm{E} 2 \mathrm{~F} 4$ has been shown to modulate the $\mathrm{G} 2 / \mathrm{M}$ checkpoint by regulating target gene expression (27). E2F target genes include genes that are involved in chromosome segregation, chromatin assembly/condensation and the mitotic spindle checkpoint. All of these proteins play important roles in $\mathrm{G} 2 / \mathrm{M}$ phase progression. In ESCC cells, the knockdown of KPNA2 attenuated E2F1 nuclear translocation and induced G2/M cell cycle arrest. These results emphasize the possibility that the primary function of KPNA2 is mediated by E2F1 in ESCC cells.

As a protein functioning in nuclear transport, the deregulation of KPNA2 may affect cargo protein translocation in cancer, thus promoting carcinogenesis. Many cancer-associated proteins have been identified as cargo proteins of KPNA2, including Chk2 (28), BRCA1 (29), NBS1 (30), RAC-1 (31), c-myc and P53 (21). A proteomic approach was previously adopted to find new cargo proteins of KPNA2 in lung cancer. Of particular interest in that investigation was $\mathrm{E} 2 \mathrm{~F} 1$, since both E2F1 nuclear translocation and its target gene expression are inhibited by KPNA2 knockdown. The E2F1-mediated signaling pathways were the most disrupted after KPNA2 knockdown. E2F1 is also regulated by KPNA2 in ESCC cells. The expression of E2F1 was higher and correlated with a poor prognosis in ESCC (32). Further analysis of the relationship between E2F1 and KPNA2 in ESCC tissues will provide definitive insight into the mechanism of KPNA2-mediated carcinogenesis and may suggest a new strategy for ESCC therapy.

In summary, the expression of KPNA2 was higher in tissues, cell lines and sera in ESCC. The knockdown of KPNA2 expression in ESCC cells attenuated cell proliferation and survival by inducing $\mathrm{G} 2 / \mathrm{M}$ phase cell cycle arrest through the E2F1 signaling pathway. KPNA2 may be a potent marker and therapeutic target for ESCC.

\section{Acknowledgements}

We thank Dr Yulin Sun, Dr Ming Liu and Miss Fang Liu for great support. This study was supported by the National High-Tech R\&D Program (no. 2012AA020206), NSFC (nos. 31070673, 31170780, 81372591, 81321091) and BJNSFC (no. 5112012) of China.

\section{References}

1. Jemal A, Bray F, Center MM, Ferlay J, Ward E and Forman D: Global cancer statistics. CA Cancer J Clin 61: 69-90, 2011.

2. Wang GQ, Abnet CC, Shen Q, Lewin KJ, Sun XD, Roth MJ, Qiao YL, Mark SD, Dong ZW, Taylor PR and Dawsey SM: Histological precursors of oesophageal squamous cell carcinoma: results from a 13 year prospective follow up study in a high risk population. Gut 54: 187-192, 2005.

3. Kelley JB, Talley AM, Spencer A, Gioeli D and Paschal BM: Karyopherin $\alpha 7$ (KPNA7), a divergent member of the importin $\alpha$ family of nuclear import receptors. BMC Cell Biol 11: 63, 2010.

4. Stewart M: Molecular mechanism of the nuclear protein import cycle. Nat Rev Mol Cell Biol 8: 195-208, 2007.

5. Goldfarb DS, Corbett AH, Mason DA, Harreman MT and Adam SA: Importin $\alpha$ : a multipurpose nuclear-transport receptor. Trends Cell Biol 14: 505-514, 2004.

6. Dahl E, Kristiansen G, Gottlob K, Klaman I, Ebner E, Hinzmann B, Hermann K, Pilarsky C, Dürst M, KlinkhammerSchalke M, Blaszyk H, Knuechel R, Hartmann A, Rosenthal A and Wild PJ: Molecular profiling of laser-microdissected matched tumor and normal breast tissue identifies karyopherin $\alpha 2$ as a potential novel prognostic marker in breast cancer. Clin Cancer Res 12: 3950-3960, 2006.

7. Dankof A, Fritzsche FR, Dahl E, Pahl S, Wild P, Dietel M, Hartmann A and Kristiansen G: KPNA2 protein expression in invasive breast carcinoma and matched peritumoral ductal carcinoma in situ. Virchows Arch 451: 877-881, 2007.

8. Gluz O, Wild P, Meiler R, Diallo-Danebrock R, Ting E, Mohrmann S, Schuett G, Dahl E, Fuchs T, Herr A, Gaumann A, Frick M, Poremba C, Nitz UA and Hartmann A: Nuclear karyopherin $\alpha 2$ expression predicts poor survival in patients with advanced breast cancer irrespective of treatment intensity. Int $\mathbf{J}$ Cancer 123: 1433-1438, 2008. 
9. Zheng M, Tang L, Huang L, Ding H, Liao WT, Zeng MS and Wang HY: Overexpression of karyopherin-2 in epithelial ovarian cancer and correlation with poor prognosis. Obstet Gynecol 116: 884-891, 2010

10. Winnepenninckx V1, Lazar V, Michiels S, Dessen P, Stas M, Alonso SR, Avril MF, Ortiz Romero PL, Robert T, Balacescu O, Eggermont AM, Lenoir G, Sarasin A, Tursz T, van den Oord JJ, Spatz A; Melanoma Group of the European Organization for Research and Treatment of Cancer: Gene expression profiling of primary cutaneous melanoma and clinical outcome. J Natl Cancer Inst 98: 472-482, 2006

11. van der Watt PJ, Maske CP, Hendricks DT, Parker MI, Denny L, Govender D, Birrer MJ and Leaner VD: The Karyopherin proteins, $\mathrm{Crm} 1$ and Karyopherin $\beta 1$, are overexpressed in cervical cancer and are critical for cancer cell survival and proliferation. Int J Cancer 124: 1829-1840, 2009.

12. Wang CI, Wang CL, Wang CW, Chen CD, Wu CC, Liang Y, Tsai YH, Chang YS, Yu JS and Yu CJ: Importin subunit alpha-2 is identified as a potential biomarker for non-small cell lung cancer by integration of the cancer cell secretome and tissue transcriptome. Int J Cancer 128: 2364-2372, 2011.

13. Gousias K, Becker AJ, Simon M and Niehusmann P: Nuclear karyopherin a2: a novel biomarker for infiltrative astrocytomas. J Neurooncol 109: 545-553, 2012.

14. Mortezavi A, Hermanns T, Seifert HH, Baumgartner MK, Provenzano M, Sulser T, Burger M, Montani M, Ikenberg K, Hofstädter F, Hartmann A, Jaggi R, Moch H, Kristiansen G and Wild PJ: KPNA2 expression is an independent adverse predictor of biochemical recurrence after radical prostatectomy. Clin Cancer Res 17: 1111-1121, 2011.

15. Yoshitake K, Tanaka S, Mogushi K, Aihara A, Murakata A, Matsumura S, Mitsunori Y, Yasen M, Ban D, Noguchi N, Irie T, Kudo A, Nakamura N, Tanaka H and Arii S: Importin- $\alpha 1$ as a novel prognostic target for hepatocellular carcinoma. Ann Surg Oncol 18: 2093-2103, 2011.

16. Jensen JB1, Munksgaard PP, Sørensen CM, Fristrup N, Birkenkamp-Demtroder K, Ulhøi BP, Jensen KM, Ørntoft TF and Dyrskjøt L: High expression of karyopherin- $\alpha 2$ defines poor prognosis in non-muscle-invasive bladder cancer and in patients with invasive bladder cancer undergoing radical cystectomy. Eur Urol 59: 841-848, 2011

17. Sakai M, Sohda M, Miyazaki T, Suzuki S, Sano A, Tanaka N, Inose T, Nakajima M, Kato $\mathrm{H}$ and Kuwano $\mathrm{H}$ : Significance of karyopherin- $\alpha 2$ (KPNA2) expression in esophageal squamous cell carcinoma. Anticancer Res 30: 851-856, 2010.

18. Sun Y, Mi W, Cai J, Ying W, Liu F, Lu H, Qiao Y, Jia W, Bi X, Lu N, Liu S, Qian X and Zhao X: Quantitative proteomic signature of liver cancer cells: tissue transglutaminase 2 could be a novel protein candidate of human hepatocellular carcinoma. J Proteome Res 7: 3847-3859, 2008.

19. Xu Y, Zhou L, Huang J, Liu F, Yu J, Zhan Q, Zhang L and Zhao X: Role of Smac in determining the chemotherapeutic response of esophageal squamous cell carcinoma. Clin Cancer Res 17: 5412-5422, 2011.
20. van der Watt PJ, Ngarande E and Leaner VD: Overexpression of $\mathrm{Kpn} \beta 1$ and Kpn $\alpha 2$ importin proteins in cancer derives from deregulated E2F activity. PLoS One 6: e27723, 2011.

21. Wang CI, Chien KY, Wang CL, Liu HP, Cheng CC, Chang YS, $\mathrm{Yu}$ JS and Yu CJ: Quantitative proteomics reveals regulation of karyopherin subunit alpha-2 (KPNA2) and its potential novel cargo proteins in nonsmall cell lung cancer. Mol Cell Proteomics 11: 1105-1122, 2012.

22. Ishida S, Huang E, Zuzan H, Spang R, Leone G, West M and Nevins JR: Role for E2F in control of both DNA replication and mitotic functions as revealed from DNA microarray analysis. Mol Cell Biol 21: 4684-4699, 2001.

23. Zhu W, Giangrande PH and Nevins JR: E2Fs link the control of G1/S and G2/M transcription. EMBO J 23: 4615-4626, 2004.

24. Dong QM, Zhang JQ, Li Q, Bracher JC, Hendricks DT and Zhao XH: Clinical significance of serum expression of GRO $\beta$ in esophageal squamous cell carcinoma. World J Gastroenterol 17: 2658-2662, 2011.

25. Quensel C, Friedrich B, Sommer T, Hartmann E and Kohler M: In vivo analysis of importin $\alpha$ proteins reveals cellular proliferation inhibition and substrate specificity. Mol Cell Biol 24: 10246-10255, 2004.

26. Huang L, Wang HY, Li JD, Wang JH, Zhou Y, Luo RZ, Yun JP, Zhang Y, Jia WH and Zheng M: KPNA2 promotes cell proliferation and tumorigenicity in epithelial ovarian carcinoma through upregulation of c-Myc and downregulation of FOXO3a. Cell Death Dis 4: e745, 2013.

27. Ren B, Cam H, Takahashi Y, Volkert T, Terragni J, Young RA and Dynlacht BD: E2F integrates cell cycle progression with DNA repair, replication, and $\mathrm{G}_{2} / \mathrm{M}$ checkpoints. Genes Dev 16 : 245-256, 2002.

28. Zannini L, Lecis D, Lisanti S, Benetti R, Buscemi G, Schneider C and Delia D: Karyopherin- $\alpha 2$ protein interacts with Chk 2 and contributes to its nuclear import. J Biol Chem 278: 42346-42351, 2003.

29. Narod SA and Foulkes WD: BRCA1 and BRCA2: 1994 and beyond. Nat Rev Cancer 4: 665-676, 2004.

30. Tseng SF, Chang CY, Wu KJ and Teng SC: Importin KPNA2 is required for proper nuclear localization and multiple functions of NBS1. J Biol Chem 280: 39594-39600, 2005.

31. Sandrock K, Bielek H, Schradi K, Schmidt G and Klugbauer N: The nuclear import of the small GTPase Rac1 is mediated by the direct interaction with karyopherin $\alpha 2$. Traffic 11: 198-209, 2010.

32. Ebihara Y, Miyamoto M, Shichinohe T, Kawarada Y, Cho Y, Fukunaga A, Murakami S, Uehara H, Kaneko H, Hashimoto H, Murakami Y, Itoh T, Okushiba S, Kondo S and Katoh H: Overexpression of E2F-1 in esophageal squamous cell carcinoma correlates with tumor progression. Dis Esophagus 17: 150-154, 2004. 